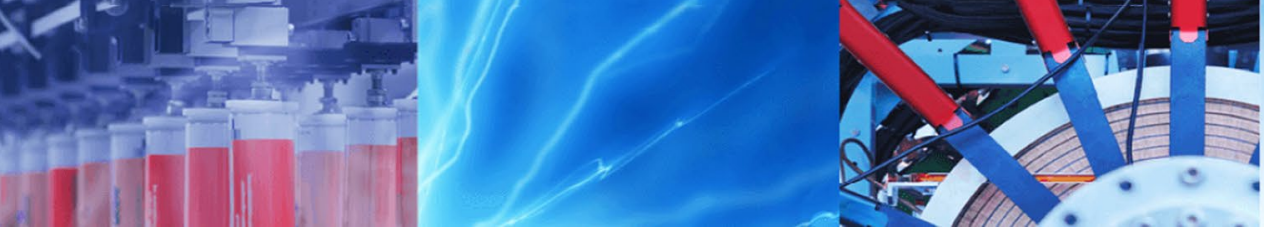

Research Article

\title{
Using locally adjustable hand-eye calibrations to reduce robot localization error
}

\author{
Marek Franaszek $^{1}$ (D) Geraldine S. Cheok ${ }^{1}$
}

Received: 5 November 2019 / Accepted: 30 March 2020 / Published online: 7 April 2020

(c) This is a U.S. Government work and not under copyright protection in the U.S.; foreign copyright protection may apply 2020

\begin{abstract}
Accurate pose of a robot end effector is required in many applications. Typically, this is achieved by robot calibration and then, registration of the robot frame to the world frame. In this paper, the registration of a poorly calibrated robot to a world frame was performed using many local hand-eye registrations and using one global registration. Both approaches were evaluated using a set of target poses. The use of the properly chosen local transformation applied to each target led to a tenfold reduction in the orientation and position error in comparison with a use of one global transformation. The median orientation error was reduced to $0.029^{\circ}$ and the median position error was reduced to $0.221 \mathrm{~mm}$ which is approximately four times larger than robot specified position repeatability.
\end{abstract}

Keywords Robot localization error · Error compensation · Forward kinematic · Hand-eye calibration · Accuracy · Repeatability

\section{Introduction}

Robot manipulators are characterized by very good repeatability. For example, the unidirectional position repeatability of the non-calibrated industrial robot was found to be better than $\pm 37 \mu \mathrm{m}$ and the orientation repeatability was at worst $\pm 87 \mu \mathrm{rad}$ [1]. Unfortunately, the accuracy of robots is frequently larger than the repeatability by about two orders of magnitude. This negatively impacts the ability of these robots to perform manufacturing tasks which require accurate knowledge of the pose of the end effector. For example, in the aerospace industry, tasks such as automated drilling require errors less than $0.25 \mathrm{~mm}$ [2].

The localization error of industrial robots can be traced back to two root causes: (1) kinematic error caused by incorrect values of the Denavit-Hartenberg (DH) parameters in the kinematic model of the serial manipulator; (2) non-kinematic errors such as elastic deformation under gravity, thermal effects, backlash, joint compliance, encoder resolution, or wear and tear of motors [3]. Out of four $\mathrm{DH}$ parameters characterizing each revolute joint, incorrect values of the joint angle zero offsets are responsible for the largest component of the total kinematic error [4]. This type of error can be substantially reduced by remastering the robot and many different calibration techniques using different sensors have been developed [5-11]. The remaining residual part of the total localization error is usually attributed to non-kinematic errors. These errors are difficult to reduce [12], and they degrade robot performance.

Different approaches have been used to compensate for the localization error and to improve robot performance. Whenever conditions allow (e.g., space and visibility) visual servoing offers great improvement in robot accuracy $[13,14]$. This type of technique is practically limited only by the accuracy of the vision system used to guide the robot. However, it requires constant tracking of the end effector relative to the final destination point, which may be problematic in some applications, e.g., robotic surgery

Marek Franaszek, marek@nist.gov| ${ }^{1}$ National Institute of Standards and Technology, Gaithersburg, MD 20899, USA. 
[15]. Active compliance control based on feedback from force/torque ( $\mathrm{F} / \mathrm{T}$ ) sensors is another technique commonly used in part assembly [16-18]. Active control based on external sensors was also used for automated welding of aerospace components [19]. Localization error in accurate robotic drilling was reduced to $0.1 \mathrm{~mm}$ and $0.2^{\circ}$ using an external sensor and compliance compensation [20]. Even as these active control techniques (as well as other passive or hybrid compliance strategies [21-23]) enable the completion of tasks requiring high accuracy, they could still benefit from reduced localization error. For example, a larger uncertainty of the starting point for the search of the hole center using $F / T$ sensors led to increased search time [24]. Inspection of large parts (e.g., airplane components [25]) performed by a line-of-sight sensor mounted on a robot arm, requires very accurate tracking of the sensor pose. In such scenarios, feedback from a F/T sensor is not available and other solutions have to be developed, e.g., Volumetric Error Compensation (VEC) techniques [26].

The method introduced in this paper also aims at reducing robot localization error so that the robot can perform manufacturing tasks requiring high accuracy. However, unlike other procedures mentioned earlier, our approach requires no error modeling nor dynamic tracking of the robot end effector. It requires the determination of locations in the work volume where higher accuracy is desired; the acquisition of a group of end-effector poses, by a pose measuring sensor, at those locations; and the corresponding robot joint angles for each pose measurement. Then, for each group of pose measurements, a separate handeye calibration is calculated and stored in a look-up table. During regular robot operations, commanded poses in the world frame are transformed to the robot frame using the transformation from the hand-eye calibration group that is closest to the commanded pose. A schematic diagram illustrating typical cyclic operations using global calibration and a modified cycle using local calibration is shown in Fig. 1.

The modification does not require changes inside robot controller as it merely updates the commanded pose. This strategy enables substantial reduction of both the orientation and position components of the robot error.

\section{Related work}

Many techniques that reduce localization error require constant tracking of the robot end effector, calculation of small corrections, and using them to update the commanded pose. For example, with the use of an optical coordinate measuring machine (CMM), the errors for two different FANUC robot models were reduced to $0.05 \mathrm{~mm}$ and $0.05^{\circ}$ [27]. The use of an iGPS to control robot pose

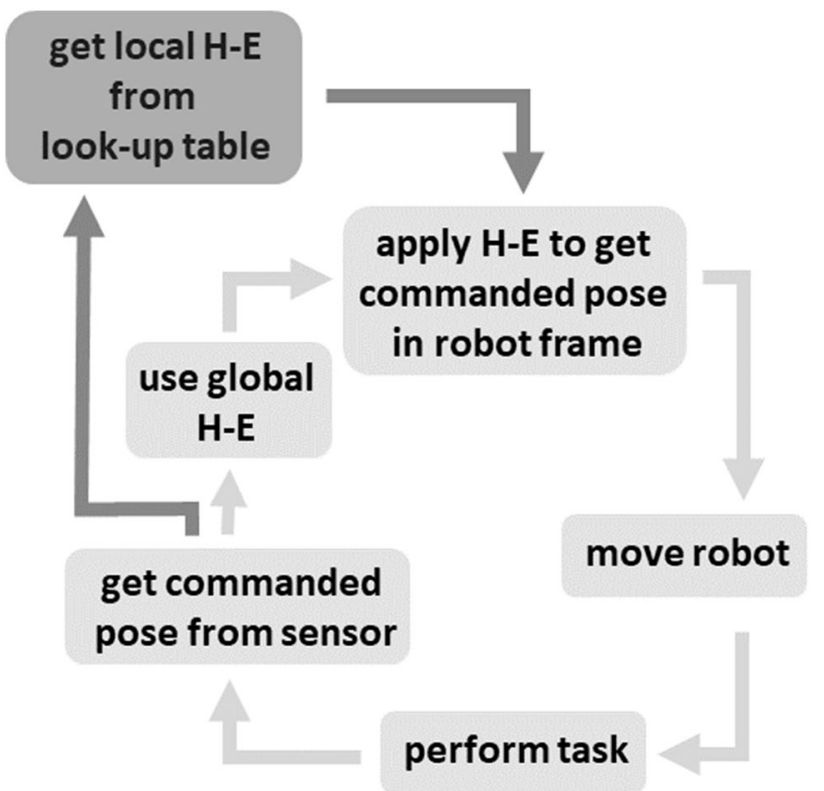

Fig. 1 Schematic diagram showing a regular automated cycle (marked in light gray) and the modified part of a cycle (marked in dark gray) which replaces global $\mathrm{H}-\mathrm{E}$ with local $\mathrm{H}-\mathrm{E}$

allowed a tenfold reduction (when compared with no control tests) of robot error, up to approximately $0.15 \mathrm{~mm}$ and $0.02^{\circ}[28]$.

Other techniques do not require constant tracking of the robot end effector. Corrections to the robot pose are initially calculated for many poses throughout the robot work volume. The calculations are based on data acquired with an external sensor and a robot. Then, during regular robot operations, corrections to the target points (i.e., points which are known only in the sensor/world coordinate frame, but which are needed in the robot frame) are interpolated from previously calculated corrections. Different interpolation methods have been used. Trilinear, cubic, and fuzzy interpolation were tested in computer simulations [29]. The kriging procedure, another interpolation technique frequently used in geostatistics [30], was applied to reduce position error of a drilling end effector mounted on a KUKA KR-210 robot arm. A spherically mounted retro-reflector (SMR) was installed on the end effector and its position was measured with a Leica AT-901 laser tracker. The average position error was reduced to $0.106 \mathrm{~mm}$ (robot repeatability was $\pm 0.06 \mathrm{~mm}$ ) [31]. A similar procedure was applied to reduce the position error of a drilling and riveting system implemented on a KUKA KR 500-3 robot arm, which has a repeatability of $\pm 0.08 \mathrm{~mm}$. The reported maximum absolute position error was reduced to $0.32 \mathrm{~mm}$ [32]. In both studies, the compensation was based on an error similarity concept (similar robot arm configurations should yield similar robot 
localization errors) and experimentally determined semivariograms [33]. No attempt to reduce orientation error was made in either study.

\section{Methodology}

Performance of local or global calibrations was gauged by the metrics defined in this section. In fixtureless applications where there is a constant offset $\hat{\boldsymbol{X}}$ between the last link frame (LLF) of the robot's kinematic chain and the end effector frame (EEF), registration between the robot base frame (RBF) and the world or sensor frame (SF) requires hand-eye (robot-sensor) calibration [34]. This requires the collection of Six Degree of Freedom (6DOF) data $\widehat{\boldsymbol{S}}_{j}$ with an external sensor so the offset $\widehat{\boldsymbol{X}}$ and $\hat{\boldsymbol{Y}}$ transformation from $\mathrm{SF}$ to $\mathrm{BRF}$ can be obtained by minimizing the differences between the poses perceived by the sensor and poses $\widehat{\boldsymbol{R}}_{j}$ derived from the robot forward kinematic model

$$
\hat{\boldsymbol{R}}_{j} \hat{\boldsymbol{X}} \approx \hat{\boldsymbol{Y}} \hat{\boldsymbol{S}}_{j}
$$

where $j=1, \ldots, J \geq 3$ and the homogenous matrix $\hat{\boldsymbol{X}}$ consists of rotation matrix $\boldsymbol{X}$ and translation vector $\boldsymbol{X}$

$\hat{\boldsymbol{x}}=\left[\begin{array}{cc}\boldsymbol{x}_{3 \times 3} & \boldsymbol{x}_{3 \times 1} \\ 0_{1 \times 3} & 1\end{array}\right]$

and similarly for $\widehat{\boldsymbol{Y}}, \hat{\boldsymbol{S}}_{j}$, and $\widehat{\boldsymbol{R}}_{j}$. The relationship between the coordinate frames are shown in Fig. 2. Equation (1) can be written for the orientation and position parts as

$\boldsymbol{R}_{j} \boldsymbol{X} \approx \boldsymbol{Y} \boldsymbol{S}_{j}$

$$
\boldsymbol{R}_{j} \boldsymbol{x}+\boldsymbol{r}_{j} \approx \boldsymbol{Y} \boldsymbol{s}_{j}+\boldsymbol{y}
$$

The quality of the registration is usually evaluated using a separate set of $K$ poses, different from the $J$ poses used to perform the hand-eye calibration. The orientation target error $\eta_{k}$ and the corresponding position error $p_{k}$ are calculated as

$\boldsymbol{R}\left(\eta_{k}, \boldsymbol{u}_{k}\right)=\boldsymbol{Y} \boldsymbol{S}_{k}\left(\boldsymbol{R}_{k} \boldsymbol{X}\right)^{-1}$

$p_{k}=\left\|\boldsymbol{R}_{k} \boldsymbol{x}+\boldsymbol{r}_{k}-\left(\boldsymbol{Y} \boldsymbol{s}_{k}+\boldsymbol{y}\right)\right\|$

where $k=1, \ldots, K, \boldsymbol{R}\left(\eta_{k}, \boldsymbol{u}_{k}\right)$ is the rotation in angle $\eta_{k}$ and axis $\boldsymbol{u}_{k}$ representation, $(\ldots)^{-1}$ is the matrix inverse, and $\|\ldots\|$ is the Euclidean norm. To check the degree of misalignment between the sensor and robot 6DOF data, relative rotations $(i, j)$ in the sensor and robot coordinate frames are calculated and the difference $\beta_{i, j}$ between the respective angles of rotation $\omega_{i, j}$ and $\varphi_{i, j}$ is obtained

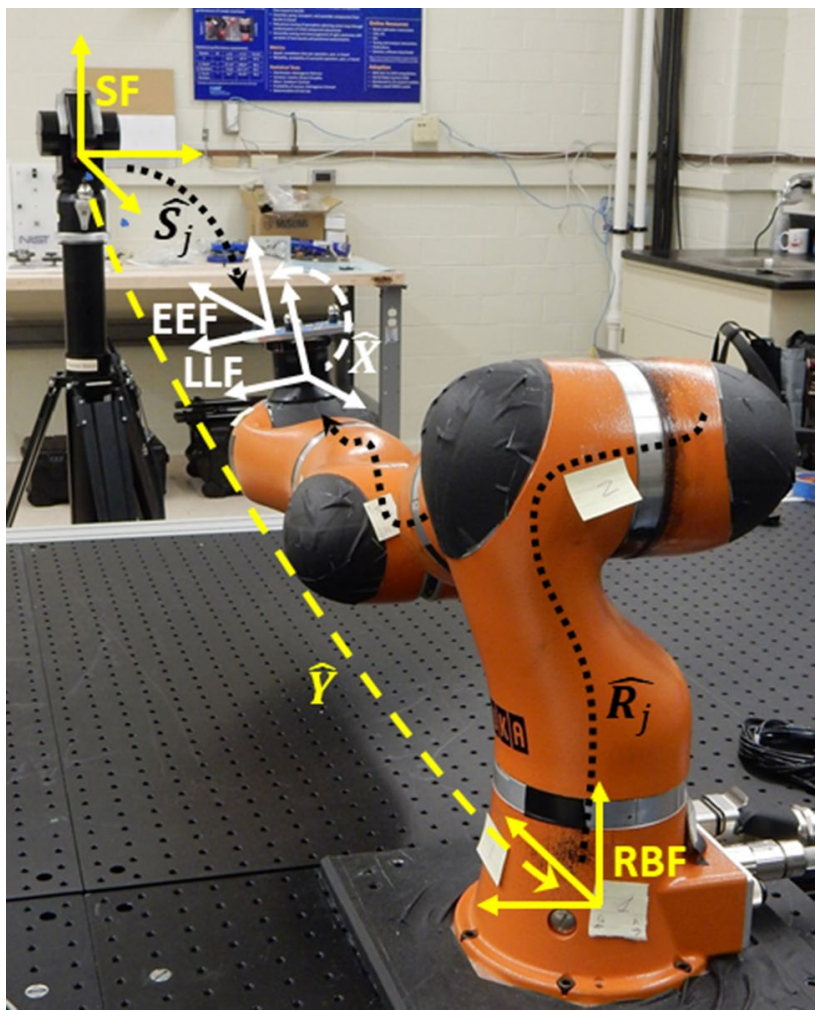

Fig. 2 Experimental setup for measuring robot pose with a laser tracker. The relationship between the four coordinate systems in the hand-eye calibration are shown

$\boldsymbol{R}\left(\omega_{i, j}, \boldsymbol{u}_{i, j}\right)=\boldsymbol{R}_{i} \boldsymbol{X}\left(\boldsymbol{R}_{j} \boldsymbol{X}\right)^{-1}=\boldsymbol{R}_{i} \boldsymbol{R}_{j}^{-1}$

$\boldsymbol{R}\left(\varphi_{i, j}, \boldsymbol{w}_{i, j}\right)=\boldsymbol{S}_{i} \boldsymbol{S}_{j}^{-1}$

$\beta_{i, j}=\varphi_{i, j}-\omega_{i, j}$

where in Eq. (5b) we used the fact that

$\operatorname{trace}\left(\boldsymbol{S}_{i} \boldsymbol{S}_{j}^{-1}\right)=\operatorname{trace}\left(\boldsymbol{Y} \boldsymbol{S}_{i} \mathbf{S}_{j}^{-1} \boldsymbol{Y}^{-1}\right)$

and that the angle of rotation $\varphi$ of the rotation matrix $\boldsymbol{R}$ is given by

$\cos (\varphi)=[\operatorname{trace}(\boldsymbol{R})-1] / 2$

Note that to use angles $\beta$ in Eq. (5c) to gauge the misalignment between the sensor and robot 6DOF data, no hand-eye calibration is needed, unlike for target errors in Eq. (4). Angles $\beta$ were used to evaluate the robot performance in different regions of its work volume while the performance of local and global hand-eye calibrations was gauged by target errors $\eta_{k}$ and $p_{k}$ defined in Eq. (4). 


\section{Experiment}

\subsection{Data collection}

A KUKA LWR 4+ collaborative, open-chain manipulator robot was used in a series of experiments. The robot was lightly used in a lab environment and not calibrated in the last three years. This 7DOF robot arm has a specified repeatability $\pm 0.05 \mathrm{~mm}$. To ensure high accuracy in Cartesian space, the stiffness of the robot was set to high.

Three $12.7 \mathrm{~mm}$ ( $0.5 \mathrm{inch}$ ) holders were glued to a plate mounted on the robot last link as shown in Fig. 3. For each robot pose, a SMR was placed in each of the three holders, and measurements were taken with an APIT3 laser tracker, where each recorded point was the average of 50 measurements. In the output file, all three SMR locations and seven joint angles of the commanded KUKA pose were stored. Then, the robot arm was moved to another pose and the data collection repeated.

The robot was commanded to 50 different target poses within its work volume. In Fig. 4, the position components $\boldsymbol{r}_{k}$ of the 6DOF target poses are plotted. In Fig. 5, the angles $\alpha_{k}$ and axes $\boldsymbol{u}_{k}$ of the orientation part $\boldsymbol{R}_{k}\left(\alpha_{k}, \boldsymbol{u}_{\boldsymbol{k}}\right)$ are shown. Since a laser tracker was used, we had to ensure that all three SMRs could be seen by the sensor. Thus, the orientation of the plate mounted on the robot arm (shown in Fig. 3) could not be in a completely arbitrary orientation.

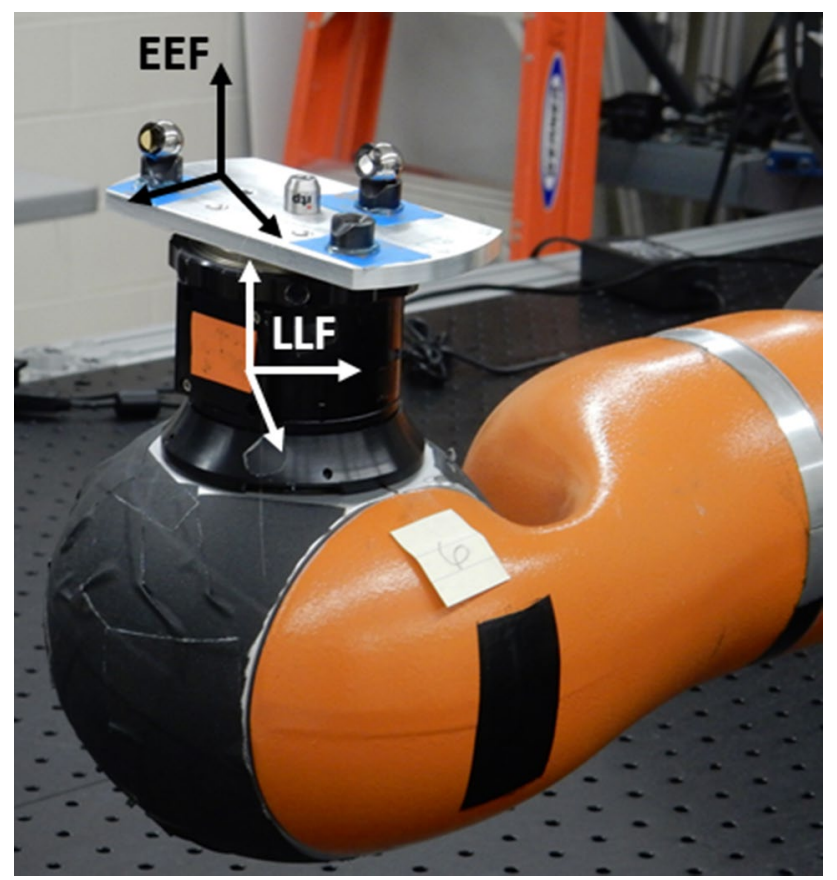

Fig. 3 Experimental setup for measuring robot pose with a laser tracker: zoom in of a plate mounted on robot arm with three holders glued to a plate and two inserted SMRs

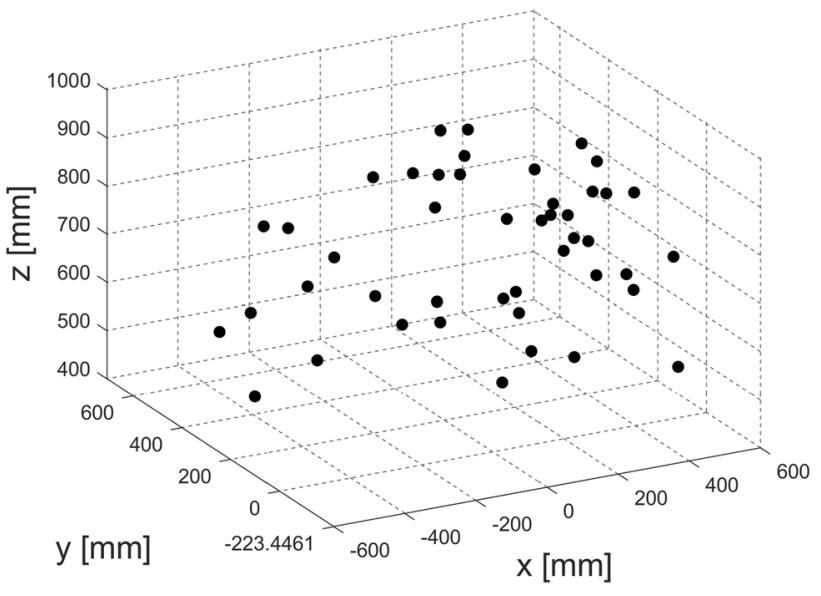

Fig. 4 Locations of 50 target poses in robot work volume

Therefore, the directions of axes of rotation in Fig. 5a are correlated.

At each target pose, the robot was commanded to move to 16 additional nearby fiducial poses, and the corresponding measurements were taken with the laser tracker. Fiducial poses close to each k-th target were obtained by changing the six joint angles of the
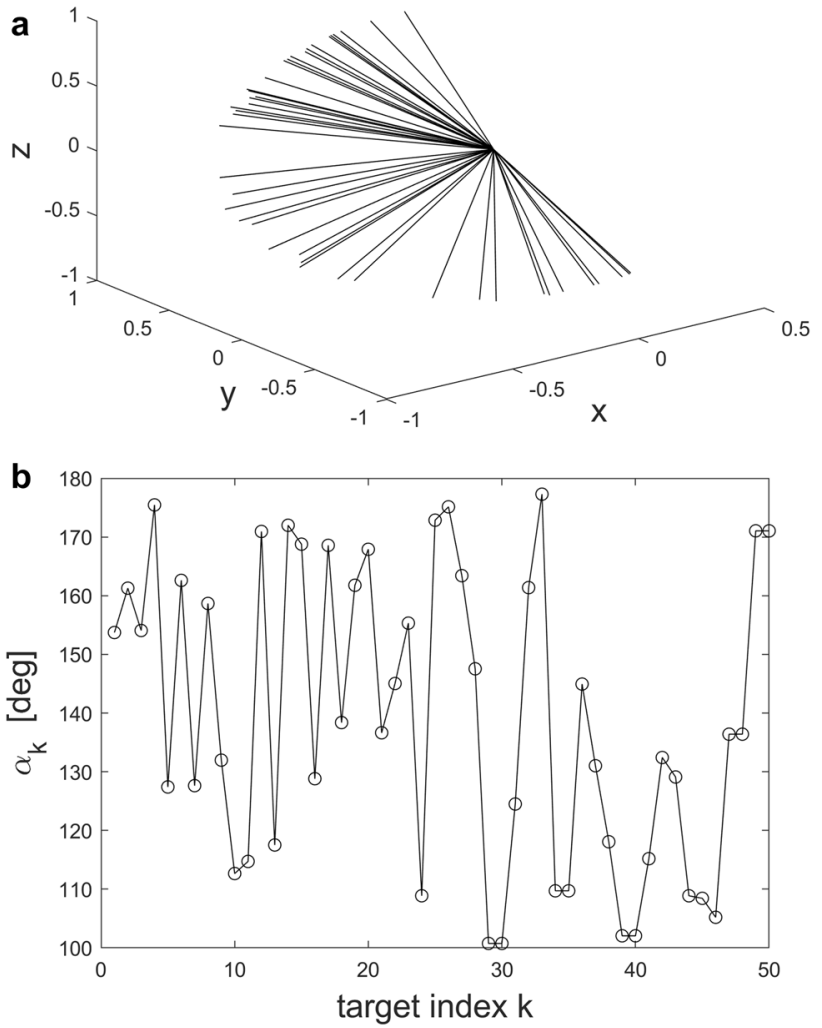

Fig. 5 Orientations of 50 target poses in robot work volume: a directions of the axes of rotation (unit vectors $\boldsymbol{u}_{k}$ ); $\mathbf{b}$ angle of rotations $\alpha_{k}$

\section{SN Applied Sciences}


target pose $\left[\vartheta_{1}, \vartheta_{2}, \vartheta_{4}, \vartheta_{5}, \vartheta_{6}, \vartheta_{7}\right]_{k}$ by a small amount $\left[2^{\circ},-2^{\circ}, \pm 2^{\circ}, \pm 3^{\circ}, \pm 3^{\circ}, \pm 4^{\circ}\right]\left(\vartheta_{3}\right.$ was set to zero for all poses). Thus, there were $50 \times 16$ fiducial poses which were later used for hand-eye calibration. The seven robot joint angles for all $J=850$ poses (targets and fiducials) were first specified and then, using a forward kinematic model, the corresponding poses in Cartesian space were calculated. These Cartesian poses were then fed to the robot controller to execute the move.

\subsection{Data post-processing}

From the triplet of 3D points measured with the laser tracker at each robot pose, a corresponding homogenous matrix $\widehat{\boldsymbol{S}}_{j}$ was determined. The quality of the 6DOF sensor data depends on the stability of the configuration of the three measured SMRs. To gauge this quality, all three distances between the SMRs were calculated for all measured poses, and the corresponding histograms, as well as the standard deviations, were obtained. Next, all triplets were transformed to one common coordinate frame (corresponding to the first triplet) using point-based registration, and the orientation matrix $\boldsymbol{C}_{j}$ was calculated for each transformed triplet. Finally, the average orientation $\boldsymbol{C}_{a v g}$ was evaluated as in [35] and then, the deviation matrix $\Delta \boldsymbol{C}_{j}$ in angle-axis $\left(\psi_{j}, \boldsymbol{c}_{j}\right)$ representation was determined

$\Delta \boldsymbol{C}_{j}\left(\psi_{j}, \boldsymbol{C}_{j}\right)=\boldsymbol{C}_{j} \boldsymbol{C}_{\text {avg }}^{-1}$

Angles $\psi_{j}$ of relative rotations were used to characterize how well the sensor data preserve rigid-body condition: in the extreme case when the condition is perfectly obeyed, all angles $\psi_{j}$ should be zero.

Misalignment between the robot and the sensor 6DOF data was investigated by calculating the angle differences $\beta_{i, j}$ using Eq. (5c). Two different calculation strategies were applied. In the first, all $J(J-1) / 2$ unique pairs of fiducial orientations $(i, j)$ were considered where $1 \leq i<j \leq J$. For $J=800$, this corresponds to approximately $3 \times 10^{5}$ pairs. In the second strategy, a pair of orientations $(k, j)$ consisted of one k-th target orientation and one of its 16 nearby fiducial orientations. The total number of such pairs was J. For each k-th target, the standard deviation $\sigma_{k}$ of angles $\beta_{k, j}$ processed in the second strategy was calculated.

Hand-eye calibration was calculated using two similar strategies. In the first approach, one global transformation $\left(\hat{\boldsymbol{X}}_{0}, \hat{\boldsymbol{Y}}_{0}\right)$ was obtained using all $J=800$ pairs of fiducial poses $\left(\widehat{\boldsymbol{R}}_{j}, \widehat{\boldsymbol{S}}_{j}\right)$ in Eq. (1). In the second strategy, $K=50$ local transformations $\left(\widehat{\boldsymbol{X}}_{k}, \hat{\boldsymbol{Y}}_{k}\right)$ were calculated from the group of 16 fiducial poses around each k-th target pose. Then, for both strategies, target registration errors $\eta_{k}$ and $p_{k}$ were calculated from Eq. (4) using either global $\left(\hat{\boldsymbol{X}}_{0}, \hat{\boldsymbol{Y}}_{0}\right)$ or local
$\left(\hat{\boldsymbol{X}}_{k}, \hat{\boldsymbol{Y}}_{k}\right)$ transformation for each $\mathrm{k}$-th target. All hand-eye transformations were calculated using the Kronecker product [36].

Deviations of the local transformations from the global one were investigated by calculating

$\boldsymbol{R}\left(\kappa_{X}(k), \boldsymbol{v}_{k}\right)=\boldsymbol{X}_{k} \boldsymbol{X}_{0}^{-1}$

$\boldsymbol{R}\left(\kappa_{Y}(k), \boldsymbol{a}_{k}\right)=\boldsymbol{Y}_{k} \boldsymbol{Y}_{0}^{-1}$

where $\kappa_{X}(k)$ is the angle of rotation gauging the difference between the global $\boldsymbol{X}_{0}$ and k-th local offset rotation $\boldsymbol{X}_{k}$. Similarly, angle $\kappa_{Y}(k)$ was used to gauge the difference between the global $\boldsymbol{Y}_{0}$ and k-th local rotation $\boldsymbol{Y}_{k}$. For the translation components, the following metrics were used for the offset and sensor to robot base transformations, respectively

$\Delta x_{k}=\left\|x_{k}-x_{0}\right\|$

$\Delta y_{k}=\left\|\boldsymbol{y}_{k}-\boldsymbol{y}_{0}\right\|$

\subsection{Simulation}

For a few targets and their associated 16 fiducial poses in Cartesian space, the inverse kinematic problem was solved and all joint angles corresponding to each pose were calculated. To get unambiguous solutions, the third joint angle $\vartheta_{3}$ was set to zero; thus, reducing the number of joints from seven to six. Then, a small constant offset $\boldsymbol{0}=\left[o_{1}, o_{2}, 0_{1} \ldots, o_{7}\right]$ was added at each pose to all joint angles $\vartheta=\left[\vartheta_{1}, \vartheta_{2}, 0, \ldots, \vartheta_{7}\right]$ yielding a new set of perturbed joint angles $\boldsymbol{\vartheta}_{p}=\boldsymbol{0}+\boldsymbol{\vartheta}$. Local hand-eye registrations $\left(\hat{\boldsymbol{X}}_{k}, \hat{\boldsymbol{Y}}_{k}\right)$ were determined as described in Sect. 4.2 where the Cartesian poses calculated from $\vartheta_{p}$ using forward kinematic served as surrogate for the uncalibrated robot poses $\widehat{\boldsymbol{R}}_{j}$ and poses calculated from $\boldsymbol{\vartheta}$ served as poses $\hat{\boldsymbol{S}}_{j}$ derived from sensor measurements, as required for determination of the hand-eye transformation in Eq. (1). Once the local registrations based on fiducials were calculated, the target orientation and position errors $\left(\eta_{k}, p_{k}\right)$ were calculated from Eq. (4) for each k-th target.

Since each Cartesian pose had up to eight possible different inverse solutions (i.e., different sets of joint angles), two different scenarios were tested: (1) the target and some or all fiducials were in different robot arm configurations; (2) the target and all its surrounding fiducials were in similar arm configurations (i.e., for each revolute joint, all 16 fiducial angles and target were similar to each other). To gauge the distance between the joint angles of the fiducials and target, the following parameter was calculated 
$\Delta \vartheta_{k}=\frac{1}{16} \sum_{n=1}^{7} \sum_{j=1}^{16}\left|\vartheta_{k}(n)-\vartheta_{k, j}(n)\right|$

where $\vartheta_{k}(n)$ is the $n$-th joint angle for the k-th target configuration and $\vartheta_{k, j}(n)$ is the $n$-th joint angle of the $j$-th fiducial associated with $\mathrm{k}$-th target. For the second scenario where fiducials differ from target by $\left[2^{\circ},-2^{\circ}, \pm 2^{\circ}, \pm 3^{\circ}, \pm 3^{\circ}, \pm 4^{\circ}\right]$, $\Delta \vartheta_{k}=16^{\circ}$. Larger values of $\Delta \vartheta_{k}$ indicate the first scenario.

\section{Results}

In Fig. 6, histograms of the distances between the three SMRs as measured by the laser tracker in 850 poses of the robot arm are shown. The standard deviations of the three distances are $14 \mu \mathrm{m}, 25 \mu \mathrm{m}$, and $17 \mu \mathrm{m}$, and the corresponding ratios $s t d /$ mean are $0.03 \%, 0.04 \%$, and $0.02 \%$. These ratios provide estimates about the preservation of the rigid body condition based on the measurements obtained by the laser tracker in different robot poses. Figure 6 indicates that the rigid body assumption does not perfectly hold. Therefore, the orientation matrices yield a non-zero spread of angles $\psi_{j}$ as shown in Fig. 7; their median value is equal to $0.0027^{\circ}$. For better visualization, two outliers of $\psi_{j}\left(0.047^{\circ}\right.$ and $\left.0.12^{\circ}\right)$ are not included in the histogram.

In Fig. 8, histograms of angles $\beta_{i, j}$ from Eq. (5c) are calculated using the two different strategies as described earlier. The standard deviation of $\beta$ was $0.326^{\circ}$ for strategy a), i.e., for all $(i, j)$ pairs of fiducial poses, and $0.044^{\circ}$ for strategy b), i.e., for all $(k, j)$ pairs consisting of $k$-th target and
Fig. 6 Histograms of distances between the three SMRs measured in different robot poses: a shortest distance; $\mathbf{b}$ medium; $\mathbf{c}$ longest distance

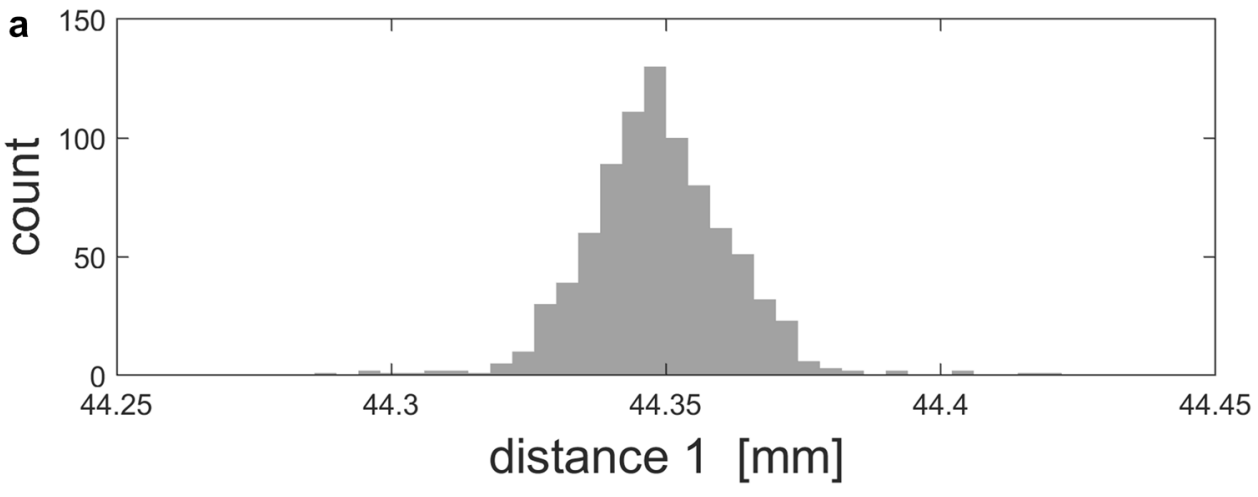

b
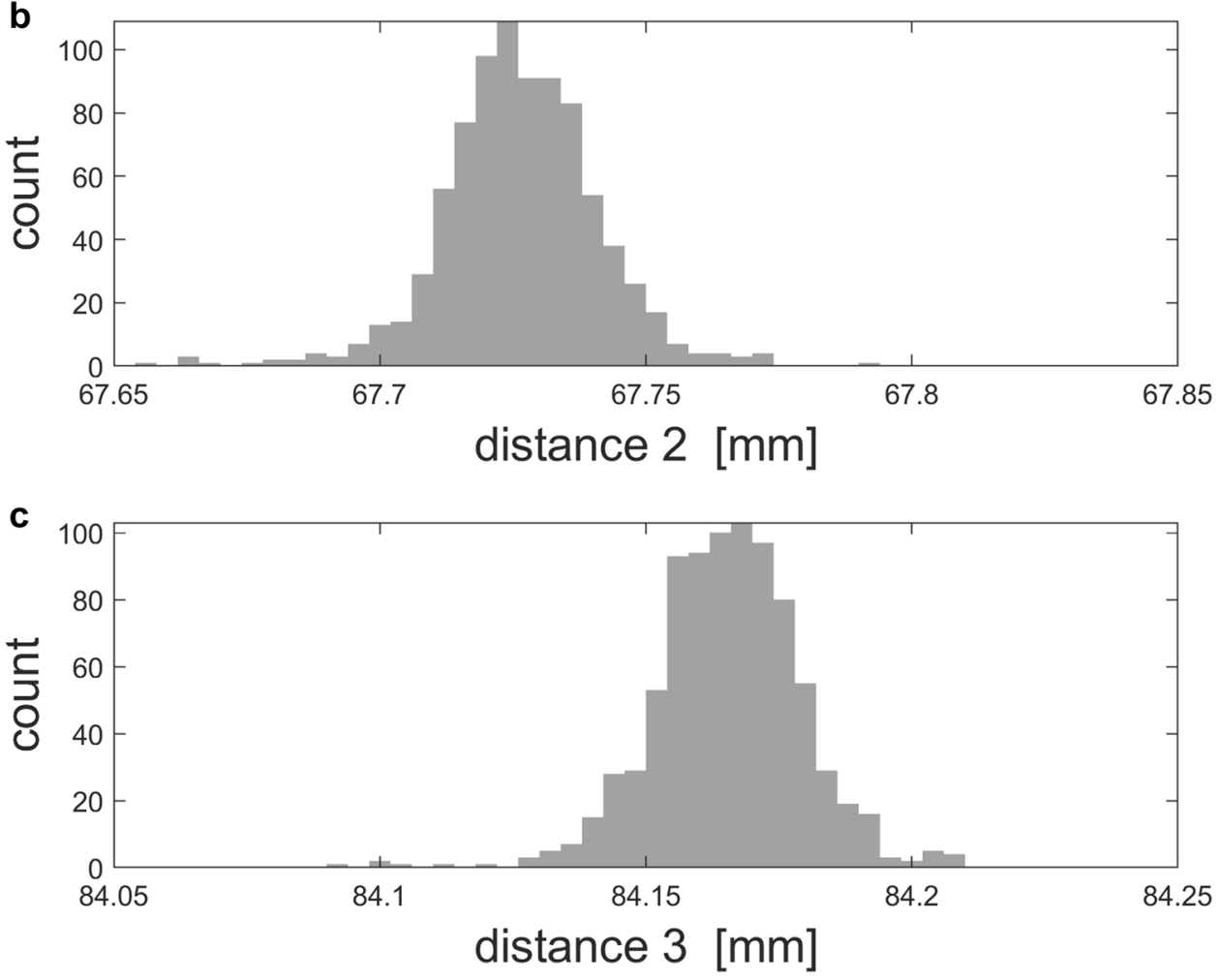


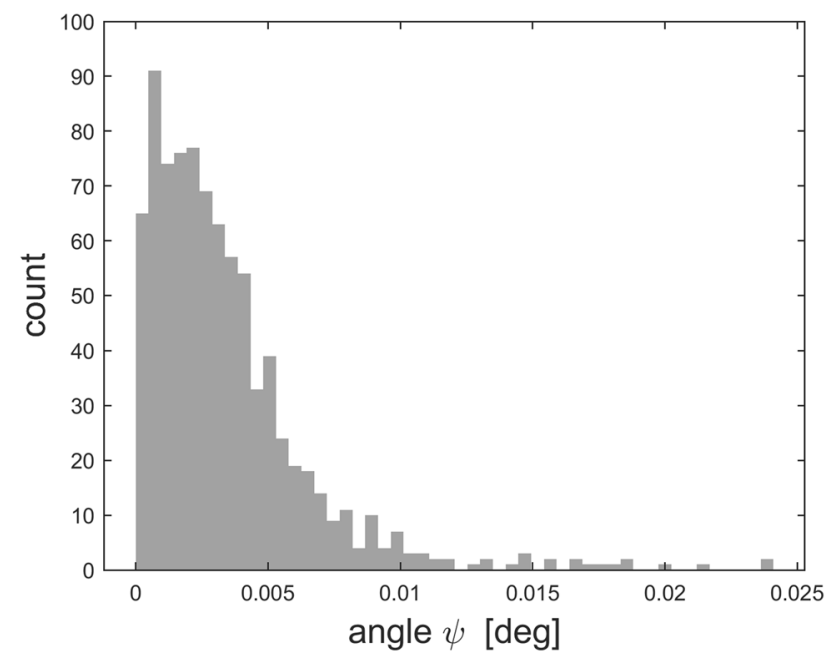

Fig. 7 Histogram of angles of relative rotations between the mean and the instantaneous rotations as determined from SMR measurements j-th fiducial. In Fig. 9, the target orientation errors $\eta_{k}$ and position errors $p_{k}$ are shown for two strategies: (1) using one, global hand-eye calibration and (2) using different local calibrations for each target. The median orientation error $\eta$ was $0.329^{\circ}$ for ( 1 ) and $0.029^{\circ}$ for (2), and the median position error $p$ was $6.13 \mathrm{~mm}$ for (1) and $0.22 \mathrm{~mm}$ for (2).

In Fig. 10, the angles $\kappa_{X}$ and $\kappa_{\gamma}$, which gauge the differences between the local and global rotational parts of the hand-eye transformation, are displayed. Similarly, the differences for the positional parts $\Delta x_{k}$ and $\Delta y_{k}$ are shown in Fig. 11 .

In Fig. 12, the relative orientation errors $\beta_{k, j}$ calculated using (5c) for strategy (2) are shown for two targets: $k=3$ and $k=48$. In Fig. 13, standard deviations $\sigma_{k}$ of errors $\beta_{k, j}$ are shown for all $K=50$ targets.

All seven targets for which the orientation error $\eta_{k}$ or position error $p_{k}$ in Fig. 9 is conspicuously larger (or, $\sigma_{k}$ in Fig. 13 is larger than $0.04^{\circ}$ ) were used as input to the simulations described in Sect. 4.3. The offset angles in vector $\boldsymbol{0}$ were set arbitrary to $\left[0.02^{\circ},-0.016^{\circ}, 0^{\circ}, 0.015^{\circ}, 0.012^{\circ},-0.17^{\circ}, 0.018^{\circ}\right]$. The results are shown in Fig. 14 where graphs plotted with
Fig. 8 Histograms of differences between angles of rotations in robot and sensor frame for: $\mathbf{a}$ all pairs $(i, j)$ of fiducial poses; $\mathbf{b}$ pairs $(k, j)$ of $k$-th target pose and $j$-th close fiducial pose
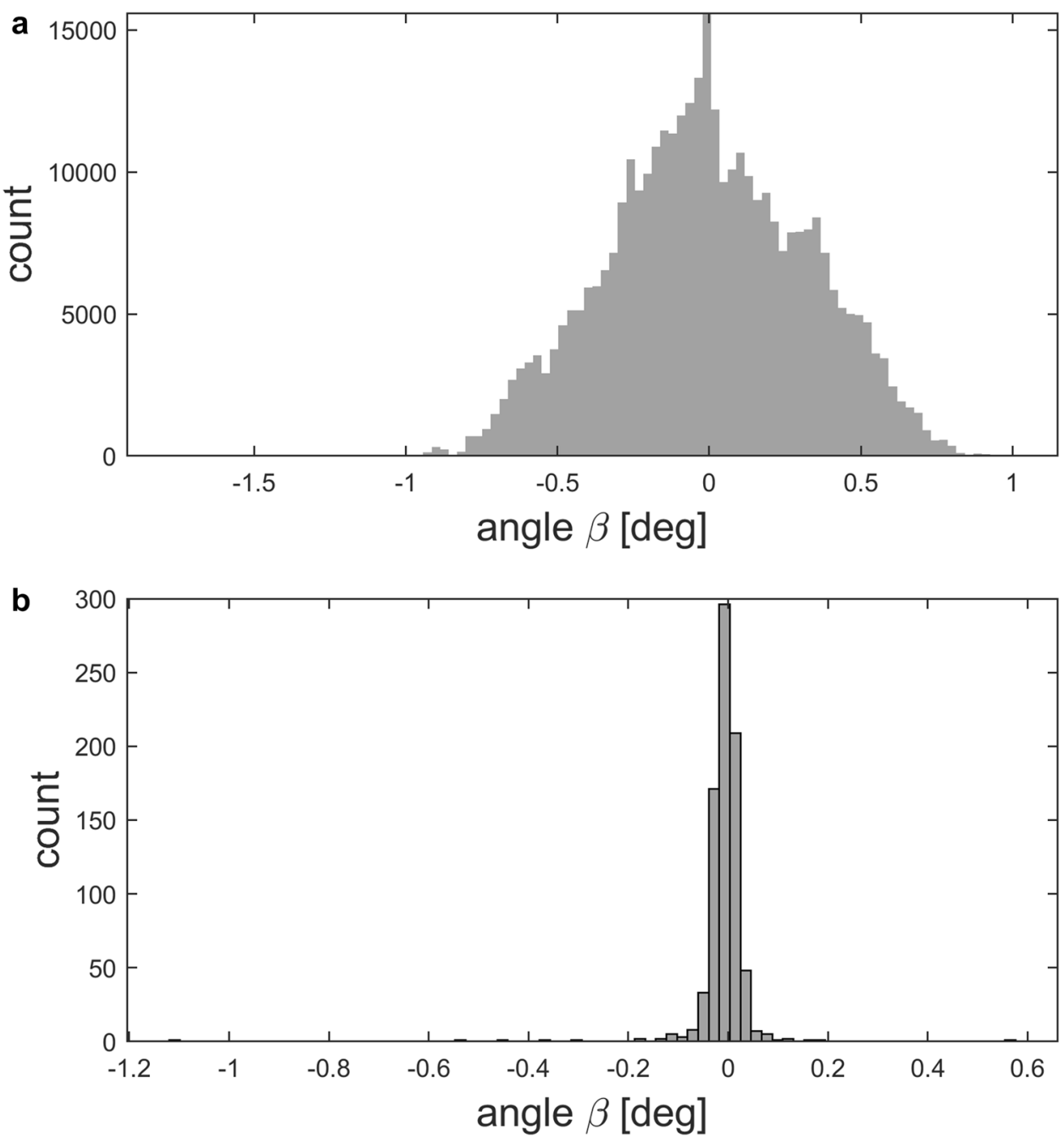
Fig. 9 Target registration errors: a orientation; $\mathbf{b}$ position, using (1) one global registration and (2) locally adjusted transformation. Filled black dots correspond to k-th targets for which either $\eta_{k}$ or $p_{k}$ have high values
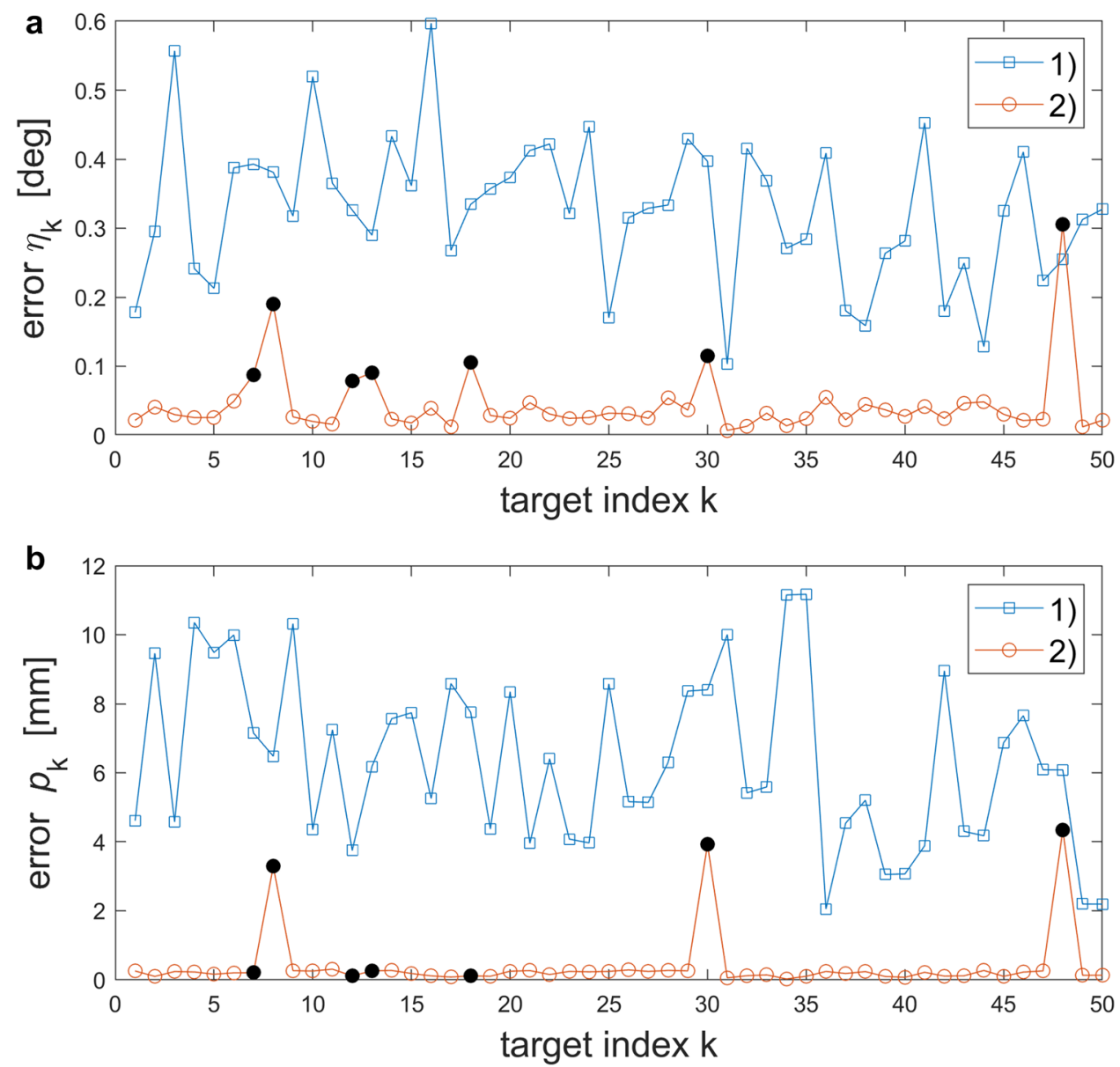

grey square symbols were obtained for the first scenario (large differences in arm configurations between target and surrounding fiducials) and graphs plotted with black dots correspond to the second scenario (small differences between target and fiducials).

\section{Discussion}

The study results clearly show that the use of multiple locally calculated hand-eye transformations $\left(\hat{\boldsymbol{X}}_{k}, \hat{\boldsymbol{Y}}_{k}\right)$ is advantageous over a use of one, global transformation $\left(\hat{\boldsymbol{X}}_{0}, \hat{\boldsymbol{Y}}_{0}\right)$. Target registration errors, obtained with local transformations, were reduced by almost an order of magnitude when compared with those where one global transformation was used. It is important to stress that the reduction was observed in both orientation errors $\eta_{k}$ shown in Fig. 9a as well as in the position errors shown in Fig. 9b. A similar error reduction was reported in $[20,27$, 28], but it was achieved by using real-time feedback from external sensors. Other techniques based on interpolation do not need dynamic tracking of robot movement (similar to our approach), but they reduced only position error [31, 32]. Equally important is to note that the reduction in both types of errors shown in Fig. 9 was achieved not only for the median values calculated from all $K=50$ targets but for each individual $k$-th target except one target (\#48). The reduced median position error $(0.221 \mathrm{~mm})$ is only four times larger than the robot specified repeatability $( \pm 0.05 \mathrm{~mm})$ per ISO 9283 standard [37]. No information for the orientation repeatability is provided in the robot's specifications. Thus, the use of the local transformations can reduce the impact of kinematic and non-kinematic robot errors. This contrasts with the use of a global transformation which requires accurate robot calibration to reduce the robot error (recall that the robot used in the experiment was not recently calibrated).

Comparison of the differences between the local $\left(\hat{\boldsymbol{X}}_{k}, \hat{\boldsymbol{Y}}_{k}\right)$ and global $\left(\hat{\boldsymbol{X}}_{0}, \hat{\boldsymbol{Y}}_{0}\right)$ transformations reveals that for the transformations $\hat{\boldsymbol{X}}$, the differences for both the rotation part gauged by $\kappa_{X}$ and for the translation part gauged by $\Delta x$ do not change much for different target locations, as shown in Figs. 10a and 11a (except for location \#48). For the transformations $\widehat{Y}$, the differences are 
Fig. 10 Differences between local and global rotations for: a hand transformation; $\mathbf{b}$ eye transformation
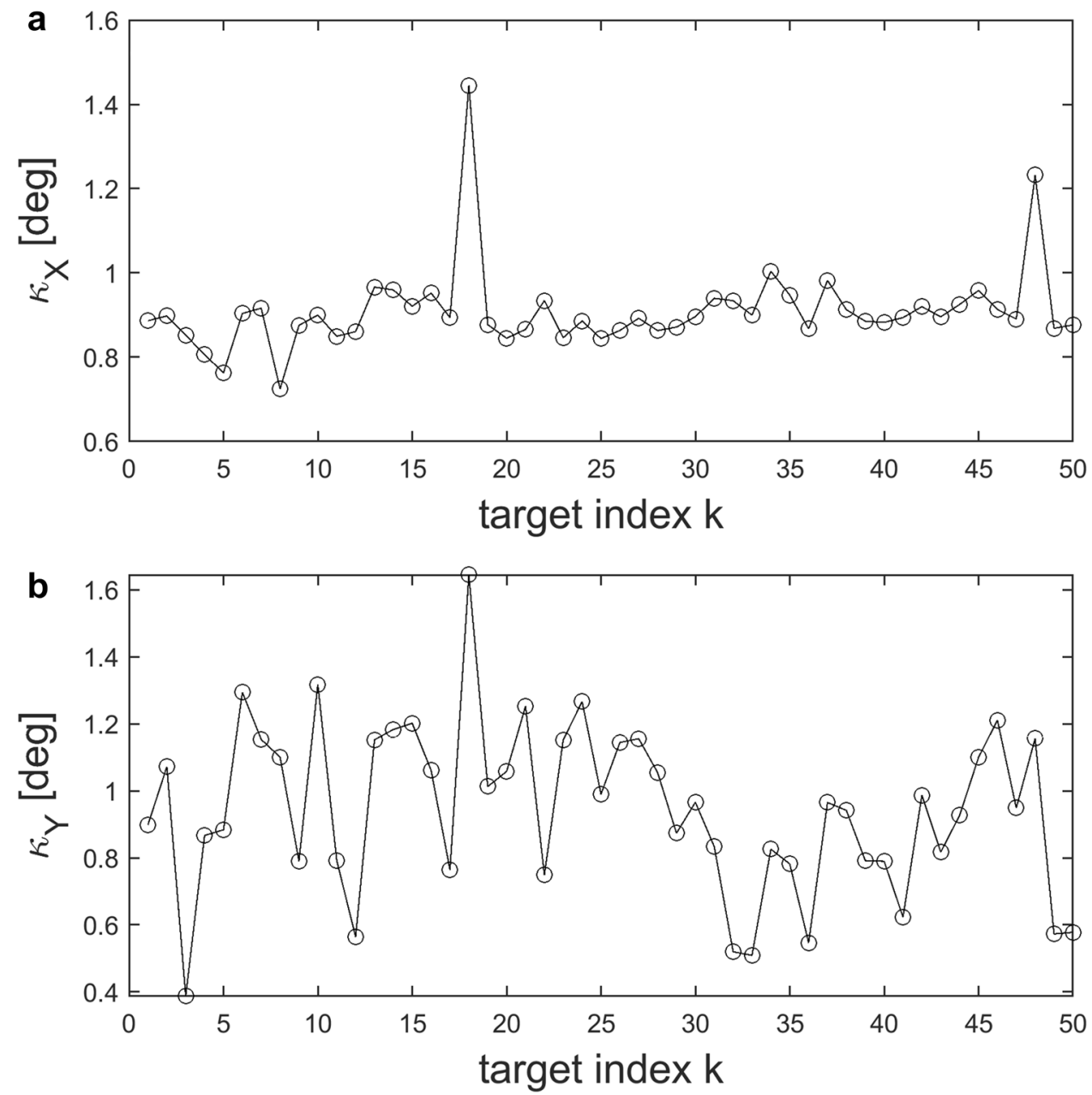

much larger and they change a lot for different locations, as shown in Figs $10 \mathrm{~b}$ and $11 \mathrm{~b}$. It is not quite clear why the local $\hat{X}_{k}$ transformations behave differently from the local $\hat{Y}_{k}$ transformations.

The results presented in this paper may seem to be intuitively obvious as the local transformations $\left(\hat{\boldsymbol{X}}_{k}, \hat{\boldsymbol{Y}}_{k}\right)$ are expected to yield smaller target errors than errors resulting from the global transformation $\left(\hat{\boldsymbol{X}}_{0}, \hat{\boldsymbol{Y}}_{0}\right)$. This expectation is supported by the distribution of angles $\beta$ calculated using Eq. (5c) which gauge the degree of misalignment between robot and sensor orientations. The angles were obtained using two different processing strategies. The histogram of $\beta$ shown in Fig. 8a corresponds to the scenario when all pairs of fiducial orientations $(i, j)$ were processed (strategy 1 ), yielding a standard deviation $\left(0.326^{\circ}\right)$ almost eight times larger than the standard deviation $\left(0.044^{\circ}\right)$ when only pairs $(k, j)$ of k-th target and its closest j-th fiducial were considered (strategy 2 ). Such a large difference between both strategies suggests that using local hand-eye calibrations $\left(\widehat{\boldsymbol{X}}_{k}, \widehat{\boldsymbol{r}}_{k}\right)$ instead of one global $\left(\widehat{\boldsymbol{X}}_{0}, \widehat{\boldsymbol{Y}}_{0}\right)$ may be advantageous. However, this intuitive reasoning may not always be valid as experiments with peg-in-hole show [38]. While the use of calculated corrections applied to the commanded robot locations (hole centers) greatly reduced the rate of failed peg insertions, there was no substantial difference in insertion rates for hole centers transformed to the robot coordinate frame using one global registration or different local registrations for individual targets. This finding in [38] conflicts with the finding in this study, and the reason for the contradiction is not clear. A possible cause is the use of 6DOF data for the hand-eye calibration and 3D data for point-based, rigidbody registration in [38].

The use of local transformations $\left(\widehat{\boldsymbol{X}}_{k}, \widehat{\boldsymbol{Y}}_{k}\right)$ instead of one global $\left(\widehat{\boldsymbol{X}}_{0}, \hat{\boldsymbol{Y}}_{0}\right)$ transformation was advantageous in all but one case where the orientation error $\eta_{k}$ was larger (for $k=48$ ), as shown in Fig. 9a. A more detailed inspection of this case revealed that the relative orientation errors $\beta_{k, j}$ calculated using Eq. (5c) for strategy 2 had unusually large 
Fig. 11 Differences between local and global translations for: $\mathbf{a}$ hand transformation; $\mathbf{b}$ eye transformation
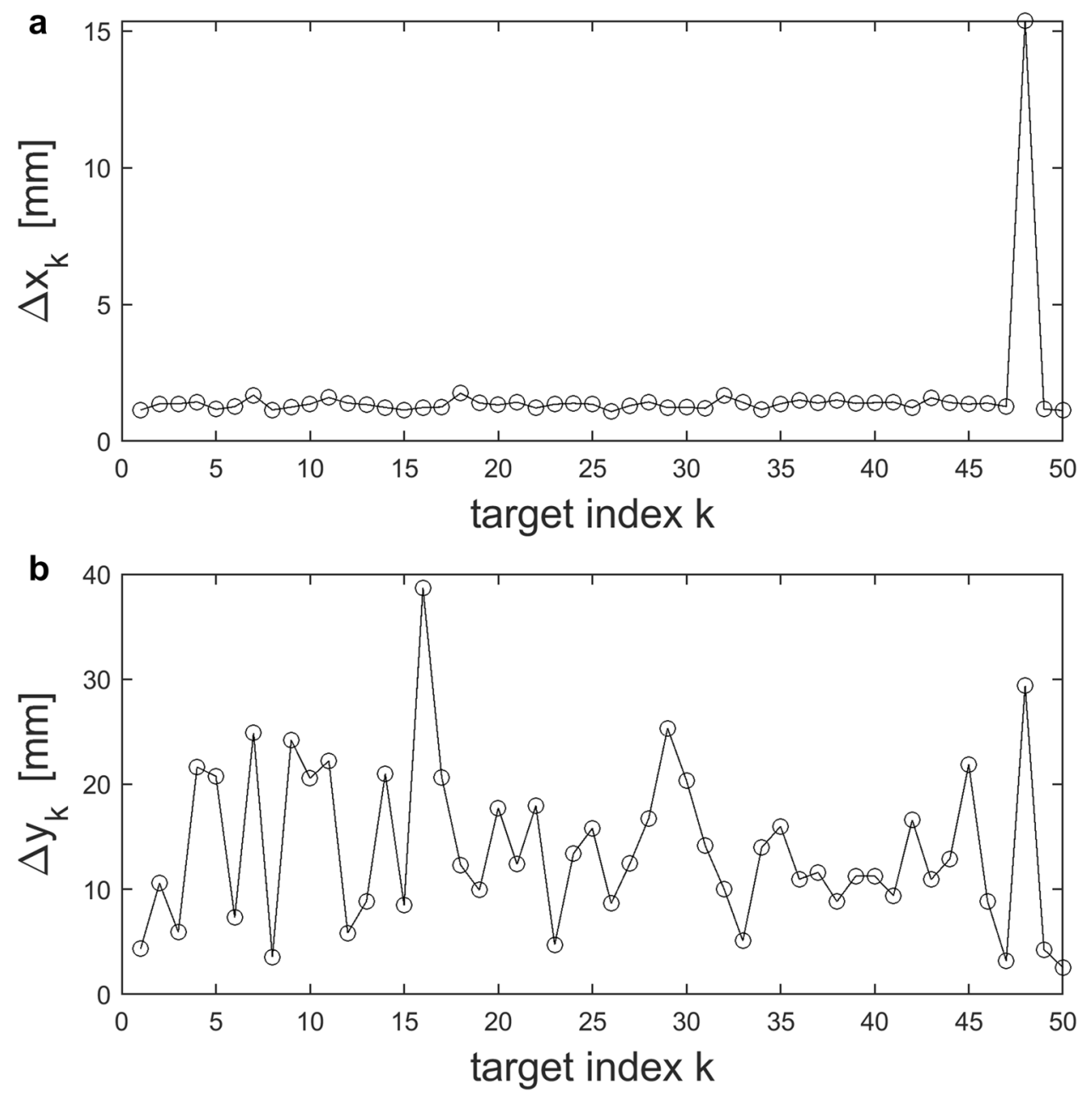

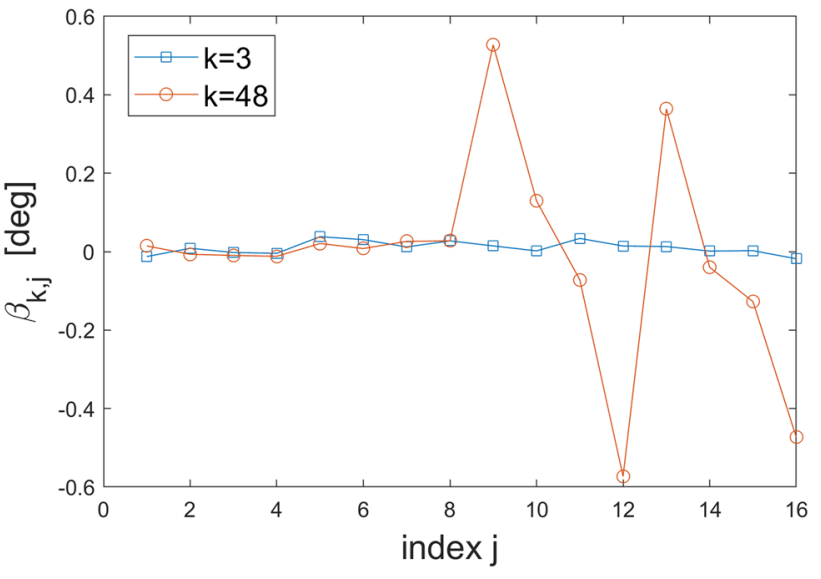

Fig. 12 Relative orientation errors of fiducials surrounding two targets: $k=3$ and $k=48$

variations when compared to other targets. The typical level of variations is displayed in Fig. 12 for target \#3 together with the unusually large variations for target \#48. This is a consequence of the fact that the commanded

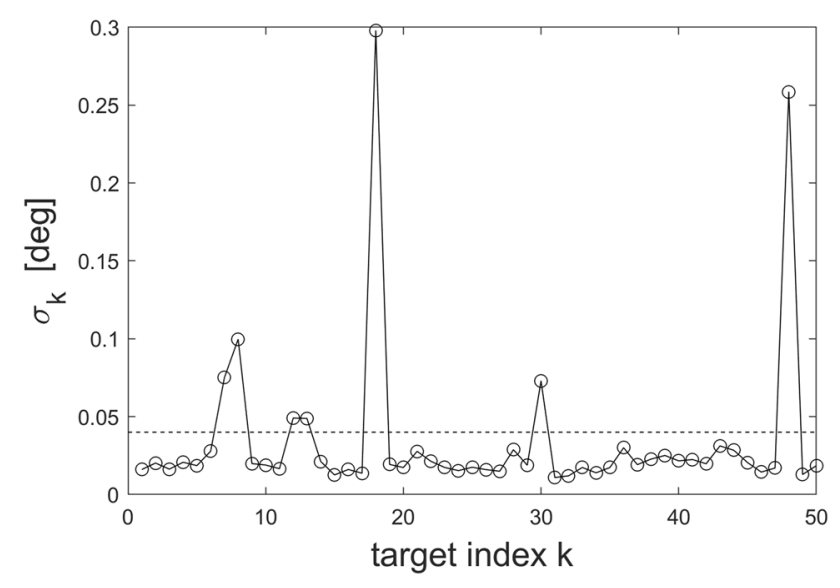

Fig. 13 Standard deviations $\sigma_{k}$ of angles $\beta_{k, j}$ (dashed line plotted at $\left.0.04^{\circ}\right)$

poses sent to the robot controller were in Cartesian space. For most target poses, 16 nearby fiducial poses were acquired in arm configurations similar to the target arm configuration. However, for some targets, even though the 
Fig. 14 Simulations results: a target orientation error; $\mathbf{b}$ target position error; $\mathbf{c}$ difference between target and fiducial joint angles. Grey squares are for the target and fiducials with different arm configurations and black dots are target and fiducials with similar arm configurations
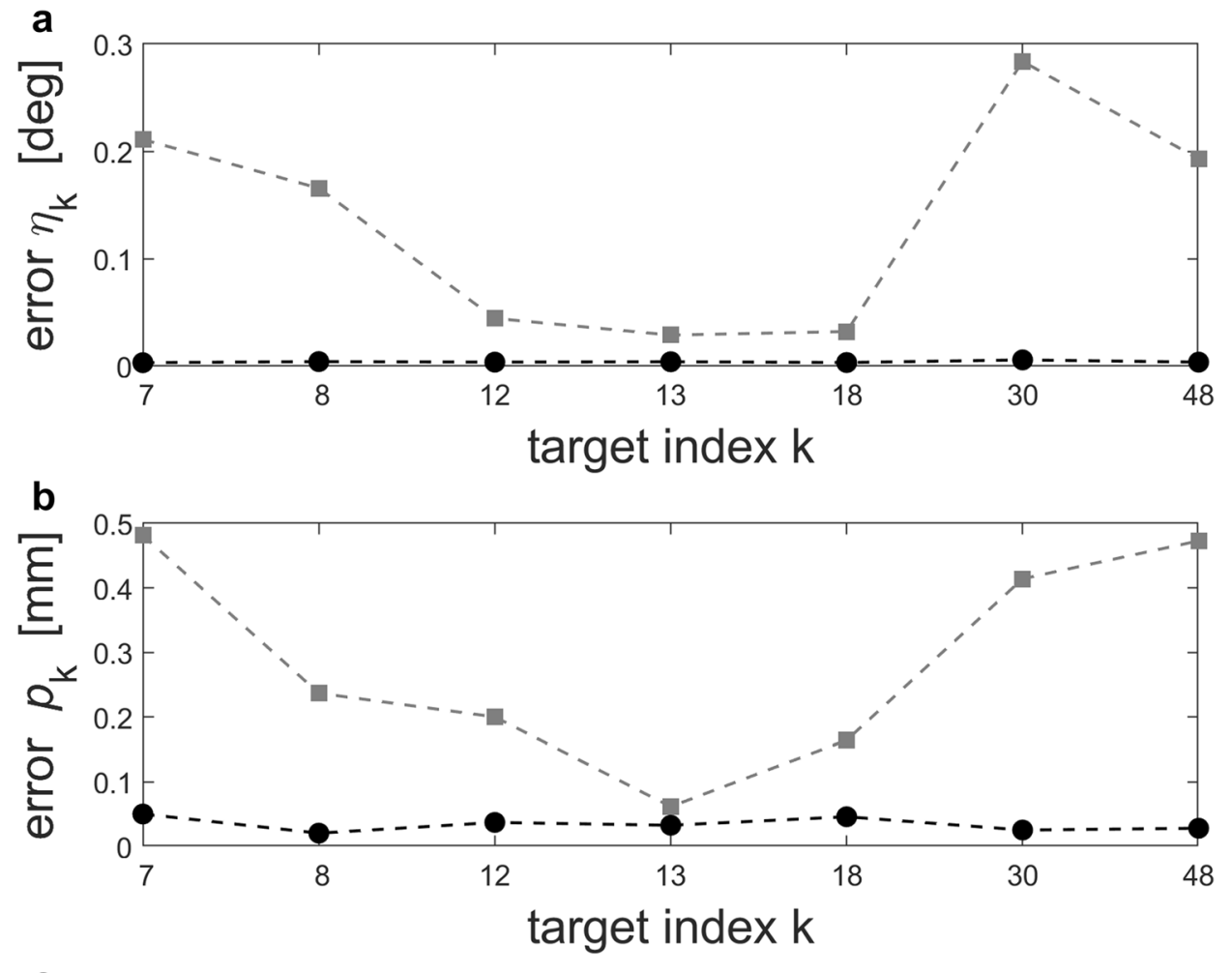

c

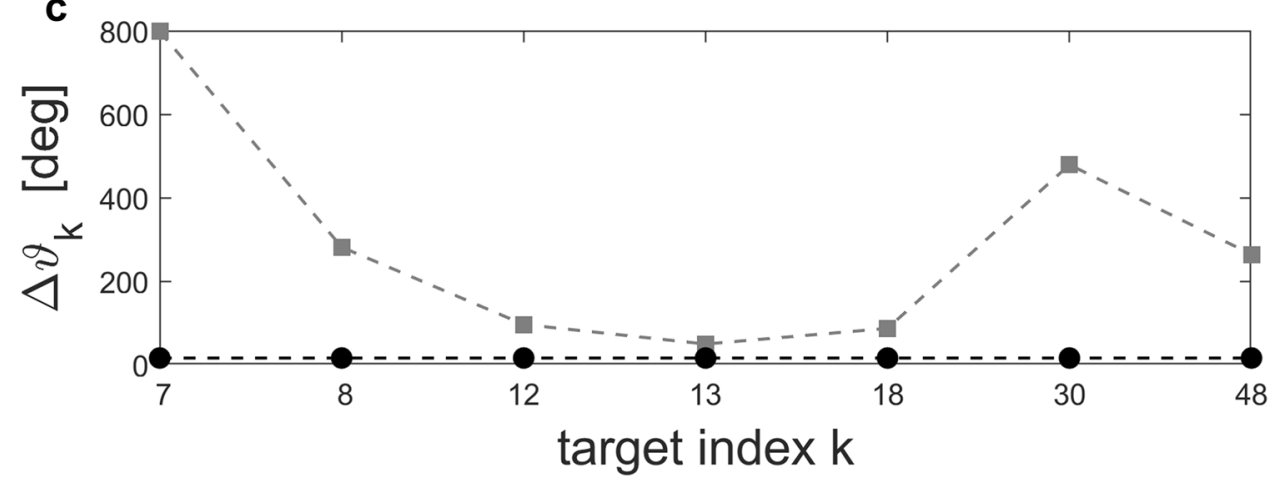

surrounding fiducial poses were close to the target pose in Cartesian space, the resulting joint angles, as determined by the robot controller using inverse kinematics, were quite different from the target configuration. Since the robot was not calibrated, the resulting poses were different from the planned Cartesian poses and this led to large variations in $\beta_{48, j}$ displayed in Fig. 12 and ultimately, a larger target orientation error $\eta_{48}$ calculated for the local transformation $\left(\widehat{\boldsymbol{X}}_{48}, \widehat{\boldsymbol{Y}}_{48}\right)$. Similar situations also occurred for other target locations for which calculated standard deviations $\sigma_{k}$ were above the threshold of $0.04^{\circ}$ marked by the dashed line in Fig. 13. Note that these seven target locations correlate with locations where the target registration errors $\eta_{k}$ and/or $p_{k}$ (calculated using k-th local hand-eye transformation) had high values, as shown in Fig. 9. Simulation results shown in Fig. 14 confirm that indeed the large differences between target and nearby fiducials robot arm configurations (i.e., joint angles $\left.\left[\vartheta_{1}, \ldots, \vartheta_{7}\right]\right)$ are responsible for the poor performance of local hand-eye registration. This conclusion is based on the observed trends as the orientation $\eta_{k}$ and position $p_{k}$ errors correlated clearly with $\Delta \vartheta_{k}$ (compare grey square plots in Fig. 14a,b with grey square plot in Fig. 14c). When the target and nearby fiducials have similar arm configurations (small $\Delta \vartheta_{k}$ ), local hand-eye registration performed very well (lines with black dots). Note that the same joint angles offset $\boldsymbol{o}$ was used to generate the data for simulation in the first (large $\Delta \vartheta_{k}$ ) and the second (small $\Delta \vartheta_{k}$ ) scenario. Thus, the elevated errors observed in the experimental data and shown in Fig. 9 could be avoided by more careful monitoring of the joint angles to ensure that the target and all nearby fiducials have similar arm configurations. That 
would require providing commanded poses to robot controller not in Cartesian space but directly in joint angles.

The robot orientation is not only determined by joint angles and forward kinematic but also by residual error in the zero offsets and by non-kinematic error. Thus, the robot orientations $\boldsymbol{R}_{i}$ and $\boldsymbol{R}_{j}$ in Eq. (5a) should be replaced by $\boldsymbol{R}_{i} \boldsymbol{G}_{i}$ and $\boldsymbol{R}_{j} \boldsymbol{G}_{j}$, where the rotation matrices $\boldsymbol{G}_{i}$ and $\boldsymbol{G}_{j}$ are unknown perturbations. For $(k, j)$ pairs of rotations, the difference between joint angles of the k-th target and its nearby $j$-th fiducial is small and therefore, $\boldsymbol{G}_{k} \approx \boldsymbol{G}_{j}$ and such small difference can be mitigated by using locally calculated offset rotation $\boldsymbol{X}_{k}$ in Eq. (5a) and Eq. (4a). The concept that similar arm configurations should yield similar robot localization errors is at the core of the kriging procedure used in [31, 32]. For arbitrary pairs of rotations $(i, j)$, both arm configurations are usually very different. Therefore, $\boldsymbol{G}_{i} \neq \boldsymbol{G}_{j}$ and the angle of rotation $\omega_{i, j}$ in Eq. (5a) derived from the kinematic model and the angle of rotation $\varphi_{i, j}$ in Eq. (5b) derived from actual measurement of the end effector pose by an external sensor are different. Indeed, the histogram of angles $\beta$ (which gauge the difference between $\omega$ and $\varphi$ ) in Fig. 8 a has a much larger spread than in Fig. 8b. That the two strategies will yield different outcomes could be expected, but what was unknown prior to these experiments was the magnitude of the difference shown in Fig. 8.

Relative commanded robot poses were compared with corresponding poses determined by an external sensor. Any differences between them (as gauged by angles $\beta$ shown in Fig. 8) were attributed to robot error only and no error was associated with the sensor. To justify this assumption, the quality of the sensor data must be evaluated independently from the robot. The laser tracker in this study did not directly measure the 6DOF data. The pose was derived from raw measurements of three $3 \mathrm{D}$ points which supposedly stayed in fixed configuration (rigid-body assumption). Such determined relative poses served as ground truth for the end effector relative poses in Eq. (5). The uncertainty of the point measured by laser tracker is $\pm 25 \mu \mathrm{m}$ which is close to standard deviations of the relative distances between three SMRs shown in Fig. 6. The spread in relative distances is a clear evidence that the rigid-body assumption is not strictly obeyed. Thus, it is important to check that the error of the derived ground truth orientation is sufficiently small. The median error $\left(0.0027^{\circ}\right)$ of angles $\psi$ shown in Fig. 7 is an order of magnitude smaller than the median of the target orientation error $\eta\left(0.029^{\circ}\right.$ for strategy 2$)$ displayed in Fig. 9 a and robot relative orientation error $\beta\left(0.044^{\circ}\right.$ for strategy 2$)$ shown in Fig. $8 \mathrm{~b}$. This indicates that the orientations determined by the sensor can be used as ground truth in this study. However, some outliers of $\psi$ in Fig. 7 are larger than $0.015^{\circ}$ and, therefore, ground truth data should be used with caution.
A possible remedy would be to use a larger plate mounted on the robot arm to increase the distances between the three SMRs shown in Fig. 3.

Data collection for this study was rather labor intensive due to the choice of the particular pose measuring system which required the manual placement of an SMR in three holders. However, there are commercially available systems which provide comparably accurate pose measurement in a fully automated mode. In this study, the fiducial poses which were used to calculate the local $\left(\hat{\boldsymbol{X}}_{k}, \hat{\boldsymbol{Y}}_{k}\right)$ transformations had the joint angles deviating from the corresponding $\mathrm{k}$-th target angles by $2^{\circ}$ to $4^{\circ}$. The range of deviations roughly defines the domain of applicability for using local transformations. In practical applications, the center of the domain should be selected in the robot workspace where higher accuracy is required to accomplish a specific task. Any target pose which falls into this domain (i.e., ranges of joint angles) should have its localization error reduced. At this time, it remains an open question (which requires further study) how large this domain can be and still benefit from the use of local transformation. Larger domains are more useful since they reduce the amount of data which need to be acquired. At the same time, larger domains yield less accurate results for points which are closer to domain boundaries. The trade-offs between both factors must be evaluated individually for each particular application.

Unlike other error compensation techniques which require either constant tracking of the robot's end effector or can compensate only its position error, the strategy introduced in this paper can lead to reduction in both the position and orientation errors, and it does not require dynamic tracking and constant feedback from external sensor.

Disclaimer Certain commercial equipments are mentioned in this paper to specify the experimental procedure adequately. Such identification is not intended to imply recommendation or endorsement by the National Institute of Standards and Technology, nor does it imply that the equipment is necessarily the best available for the purpose.

\section{Compliance with ethical standards}

Conflict of interest On behalf of all authors, the corresponding author states that there is no conflict of interest.

\section{References}

1. Slamani M, Nubiola A, Bonev IA (2012) Assessment of the positioning performance of an industrial robot. Ind Robot 39(1):5768. https://doi.org/10.1108/01439911211192501 
2. Devlieg R (2010) Expanding the use of robotics in airframe assembly via accurate robot technology. SAE Int J Aerosp 3(1):198-203. https://doi.org/10.4271/2010-01-1846

3. Greenway B (2000) Robot accuracy. Ind Robot 27(4):257-265

4. Conrad K, Shiakolas P, Yih T (2000) Robot calibration issues: accuracy, repeatibility and calibration. In: 8th mediterranean conference on control and automation, Rio Patras

5. Aoyagi S, Kohama A, Nakata Y, Hayano Y, Suzuki M (2010) Improvement of robot accuracy by calibrating kinematic model using a laser tracking system-scompensation of nongeometric errors using neural networks and selection of optimal measuring points using genetic algorithm. Paper presented at the IEEE/RSJ international conference on intelligent robots and systems, Taipei

6. Elatta AY, Gen LP, Zhi FL, Daoyuan Y, Fei L (2004) An overview of robot calibration. Inf Technol J 3(1):74-78

7. Joubair A, Bonev IL (2015) Kinematic calibration of a six-axis serial robot using distance and sphere constraints. Int J Adv Manuf Technol 77:515-523. https://doi.org/10.1007/s0017 0-014-6448-5

8. Motta JMST, Carvalho GCd, McMaster RS (2001) Robot calibration using a 3D vision-based measurement system with a single camera. Robot Comput Integr Manuf 17:487-497

9. Nubiola A, Slamani M, Joubair A, Bonev IL (2014) Comparison of two calibration methods for a small industrial robot based on an optical CMM and a laser tracker. Robotica 32(3):447-466. https ://doi.org/10.1017/S0263574713000714

10. Santolaria J, Conte J, Gines M (2013) Laser tracker-based kinematic parameter calibration of industrial robots by improved CPA method and active retroreflector. Int J Adv Manuf Technol 66:2087-2106. https://doi.org/10.1007/s00170-012-4484-6

11. Wu Y, Klimchik A, Caro S, Furet B, Pashkevich A (2015) Geometric calibration of industrial robots using enhanced partial pose measurements and design of experiments. Robot Comput Integr Manuf 35:151-168. https://doi.org/10.1016/j. rcim.2015.03.007

12. Joubair A, Bonev IL (2015) Non-kinematic calibration of a six-axis serial robot using planar constraints. Prec Eng 40:325-333. https ://doi.org/10.1016/j.precisioneng.2014.12.002

13. Chaumette F, Hutchinson S (2006) Visual servo control part I: basic approaches. IEEE Robot Autom Mag 13(4):82-90

14. Chaumette F, Hutchinson S (2007) Visual servo control part II: advanced approaches. IEEE Robot Autom Mag 14(1):109-118

15. Song Y, An G, Zhang J (2009) Positioning accuracy of a medical robotic system for spine surgery. Paper presented at the 2 nd international conference on biomedical engineering and informatics BMEl'09, Tianjin

16. J.Bos, A.Wahrburg, K.D.Listmann (2017) Iteratively learned and temporally scaled force control with application to robotic assembly in unstructured environments. Paper presented at the IEEE international conference on robotics and automation (ICRA), Singapore

17. Abdullah MW, Roth $H$, Weyrich M, Wahrburg J (2015) An approach for peg-in-hole assembling using intuitive search algorithm based on human behavior and carried by sensors guided industrial robot. IFAC-PapersOnLine 48(3):1476-1481

18. Y.Xu, Y.Hu, L.Hu (2015) Precision peg-in-hole assembly strategy using force-guided robot. Paper presented at the 3rd interantional conference on machinery, materials and information technology applications (ICMMITA), Qingdao

19. Tingelstad L, Capellan A, Thomessen T, Lien TK (2012) Multirobot assembly of high-performance aerospace components. Paper presented at the 10th IFAC symposium on robot control, Dubrovnik, Croatia

20. Posada JRD, Schneider U, Pidan S, Geravand M, Stelzer P, Verl A (2016) High accurate robotic drilling with external sensor and compliance model-based compensation. Paper presented at the IEEE international conference on robotics and automation ICRA, Stockholm

21. Park H, Park J, Lee D-H, Park J-H, Baeg M-H, Bae J-H (2017) Compliance-based robotic peg-in-hole assembly strategy without force feedback. IEEE Trans Ind Electron 64(8):6299-6309

22. Polverini MP, Zanchettin AM, Castello S, Rocco P (2016) Sensorless and constraint based peg-in-hole task execution with a dual-arm robot. Paper presented at the ICRA, Stockholm

23. Luo RC, Chang A, Li Ch (2017) A novel peg-in-hole approach based on geometrical analysis for inclined uncertainty. Paper presented at the IEEE international conference on advanced intelligent mechatronics (AIM), Munich

24. Wyk KV, Culleton M, Falco J, Kelly K (2018) Comparative peg-inhole testing of a force-based manipulation controlled robotic hand. IEEE Trans Robot

25. Liu C, Li Y, Hao X (2017) An adaptive machining approach based on in-process inspection of interim machining states for largescaled and thin-walled complex parts. Int J Adv Manuf Technol 90:3119-3128. https://doi.org/10.1007/s00170-016-9647-4

26. Bristow DA, Tharayil M, Alleyne AG (2006) A survey of iterative learning control. IEEE Control Systems Magazine June 96-114

27. Gharaaty S, Shu T, Joubair A, Xie WF, Bonev IA (2018) Online pose correction of an industrial robot using an optical coordinate measure machine system. IntJ Adv Robot Syst. https://doi. org/10.1177/1729881418787915

28. Norman AR, Schonberg A, Gorlach IA, Schmitt R (2013) Validation of iGPS as an external measurement system for cooperative robot positioning. Int J Adv Manuf Technol 64:427-446. https:// doi.org/10.1007/s00170-012-4004-8

29. Bai Y, Wang D (2010) On the comparison of trilinear, cubic spline, and fuzzy interpolation methods in the high-accuracy measurements. IEEE Trans Fuzzy Syst 18(5):1016-1022

30. Myers DE (1994) Spatial interpolation: an overview. Geoderma 62:17-28

31. Chen D, Yuan P, Wang T, Ying C (2018) A compensation method based on error similarity and error correlation to enhance the position accuracy of an aviation drilling robot. Meas Sci Technol 29:1-11. https://doi.org/10.1088/1361-6501/aacd6e

32. Zeng Y, Tian W, Li D, He X, Liao W (2017) An error-similarity-based robot positional accuracy improvement method for a robotic drilling and riveting system. Int J Adv Manuf Technol 88:27452755. https://doi.org/10.1007/s00170-016-8975-8

33. Olea RA (2006) A six-step practical approach to semivariogram modeling. Stoch Env Res Risk Assess 20(5):307-318. https://doi. org/10.1007/s00477-005-0026-1

34. Zhan Q, Wang X (2012) Hand-eye calibration and positioning for a robot drilling system. Int J Adv Manuf Technol 61:691-701. https://doi.org/10.1007/s00170-011-3741-4

35. Moakher M (2002) Means and averaging in the group of rotations. SIAM J Matrix Anal Appl 24(1):1-16

36. Shah M (2013) Solving the robot-world/hand-eye calibration problem using the Kronecker product. J Mech Robot. https:// doi.org/10.1115/1.4024473

37. ISO (1998) Manipulating industrial robots: performance criteria and related test methods, vol 9283.

38. Cheok GS, Franaszek M, Wyk Kv, Marvel JA (2018) Improving automated insertion applications by restoring rigid-body condition in point-based registration. https://doi.org/10.6028/NIST. IR.8198r1

Publisher's Note Springer Nature remains neutral with regard to jurisdictional claims in published maps and institutional affiliations. 